\title{
O butorfanol na anestesia pela romifidina-tiletamina-zolazepam em gatos
}

\author{
Butorphanol in romifidine-tiletamine-zolazepam anesthesia in cats
}

\begin{abstract}
André Luis Selmi ${ }^{1}$ Glenda Ramalho Barbudo-Selmi ${ }^{1}$ Guilherme Maia Mendes ${ }^{2}$ Concepta McManus ${ }^{3}$ Christine Souza Martins ${ }^{1}$
\end{abstract}

RESUMO

Visando observar os efeitos do butorfanol $(B)$ na anestesia produzida pela associação de romifidina $(R)$ e tiletamina-zolazepam (TZ), foram utilizados seis gatos adultos, de forma que todos animais receberam a associação de romifidina-tiletamina-zolazepam (grupo $R T Z$ ) ou a associação de romifidina-tiletamina-zolazepam-butorfanol (grupo RTZB). Os animais receberam em aplicação única, por via intramuscular, $7 \mathrm{mg} . \mathrm{kg}^{-1}$ de tiletamina e $7 \mathrm{mg} \cdot \mathrm{kg}^{-1}$ de zolazepam e $40 \mu \mathrm{g} . \mathrm{kg}^{-1}$ de romifidina (grupo $R T Z$ ) ou a mesma associação acrescida de $0,2 \mathrm{mg} . \mathrm{kg}^{-1}$ de $B$ (grupo RTZB). A freqüência cardíaca, freqüência respiratória, pressão arterial sistólica, diastólica e média por método não-invasivo oscilométrico, saturação de oxihemoglobina e temperatura retal foram avaliadas durante 120 minutos e comparadas aos valores basais. Os efeitos anestésicos foram caracterizados por meio de um sistema de escores. Outros dados como período de latência, periodo anestésico hábil e período de recuperação foram mensurados para efeito comparativo. Os periodos de latência e anestésico hábil foram significativamente mais prolongados no grupo $R T Z B$. Ocorreu diminuição da freqüência respiratória no grupo $R T Z B$, havendo decréscimo transitório no grupo RTZ. A freqüência cardíaca não variou no grupo $R T Z$ até os 60 minutos e decresceu significativamente no grupo $R T Z B$. Conclui-se que a associação RTZ produz anestesia com mínimos efeitos cardiovasculares e que a adição do butorfanol à associação prolonga o tempo anestésico hábil, além de proporcionar analgesia mais duradoura, mas provoca efeitos colaterais como decréscimo da freqüencia cardíaca e da freqüencia respiratória em gatos.

\author{
Palavras-chave: romifidina, tiletamina-zolazepam, \\ butorfanol, gatos, anestesia.
}

\begin{abstract}
The effect of butorphanol was investigated in six adult cats anesthetized with romifidine-tiletaminezolazepam. Cats were given romifidine $\left(40 \mu \mathrm{g} . \mathrm{kg}^{-1}\right)$ tiletamine (7mg. $\left.\mathrm{kg}^{-1}\right)$ and zolazepam (7 $\left.\mathrm{mg} . \mathrm{kg}^{-1}\right)$ (RTZ) intramuscularly, or $R T Z$ and butorphanol $\left(0.2 \mathrm{mg} \cdot \mathrm{kg}^{-1}\right)$ $(R T Z B)$. Heart rate, respiratory rate, oscillometric systolic blood pressure, diastolic blood pressure and mean blood pressure, oxihemoglobin saturation and rectal temperature were determined for 120 minutes and compared to baseline values. Anesthetic effects were evaluated using a score system. Time of induction, anesthesia and recovery were also determined for comparison. Induction time and anesthetic time were significantly longer in RTZB. In the RTZB group a significant decrease in respiratory rate was observed while in the RTZ group this was transitory. Heart rate did not change in the RTZ group until 60 minutes and decreased significantly in the RTZB group from the time of injection. It is concluded that RTZ is an effective anesthetic combination with minimal cardiovascular side effects and that addition of butorphanol to this combination prolongs the anesthetic time and induces analgesia for a longer period, but causes a decrease in heart and respiratory rate in cats.
\end{abstract}

Key words: romifidine, tiletamine-zolazepam, butorphanol, cats, anesthesia.

\footnotetext{
${ }^{1}$ Médico Veterinário, Professor Substituto, Universidade de Brasília (UnB), Faculdade de Agronomia e Medicina Veterinária (FAV), CP 04508, 70910-970, Brasília, Distrito Federal. Email:selmi@unb.br. Autor para correspondência.

${ }^{2}$ Acadêmico. Curso de Medicina Veterinária, UnB/FAV.

${ }^{3}$ Zootecnista, Professor Adjunto, UnB/FAV.
} 


\section{INTRODUÇÃO}

Devido a sua rápida e tranqüila indução, a associação do agente dissociativo tiletamina ao benzodiazepínico zolazepam tem sido amplamente utilizada em pequenos animais, principalmente como agente pré-anestésico e em procedimentos radiográficos (ILKIW, 1992). Os efeitos sobre a função cardiovascular são mínimos, ocorrendo depressão inicial seguida pelo aumento da freqüência cardíaca, pressão arterial e resistência vascular, provavelmente resultado da ação inotrópica negativa da tiletamina e posterior aumento indireto do tono simpático (HELLYER et al., 1988; HELLYER et al., 1989; WILSON et al., 1993). Após a administração da associação, observa-se transitório decréscimo da freqüência respiratória e aumento da $\mathrm{PaCO}_{2}$ (HELLYER et al., 1988). Em gatos, a tiletamina pode rèsultar no aparecimento do padrão respiratório apnêustico que é rapidamente convertido ao padrão respiratório normal pela ação do zolazepam (CALDERWOOD et al., 1971; FAGGELLA \& ARONSOHN, 1993).

Apesar de seus mínimos efeitos deletérios, a associação tiletamina-zolazepam não proporciona satisfatória analgesia visceral, e portanto não é recomendada como agente único em procedimentos cirúrgicos (FAGGELLA \& ARONSOHN, 1993; HUBBELL, 2000). Em altas doses, promove estado semelhante à anestesia geral, normalmente correlacionada a retornos anestésicos de intensa agitação (ILKIW, 1992; HUBBELL, 2000). A utilização conjunta de agentes opióides, agonistas adrenérgicos alfa-2 e benzodiazepínicos possibilita a realização de cirurgias de curta duração em cães (TRANQUILLI et al., 1990; VERSTEGEN \& PETCHO, 1993; PYPENDOP et al., 1996). Em contrapartida, observouse que a adição de agonistas adrenérgicos alfa-2 e opióides proporciona a potencialização dos efeitos da tiletamina-zolazepam, resultando em aumento da antinocicepção (WILSON et al., 1992).

A romifidina é um potente e seletivo agonista adrenérgico alfa-2 que produz efeitos cardiorespiratórios similares aos demais agentes deste grupo (LEMKE, 1999; PYPENDOP \& VERSTEGEN, 2001). Apesar dos escassos estudos em gatos (CRUZ et al., 2000; SELMI et al., 2001), nos últimos anos, a utilização de romifidina em cães tem sido extensamente avaliada (LEMKE, 1999; PYPENDOP \& VERSTEGEN, 2001). O butorfanol, um opióide agonista-antagonista de uso freqüente na prática anestésica veterinária (HOSGOOD, 1990), produz analgesia com mínima influência sobre os parâmetros respiratórios (TRIM, 1983; KO et al., 1996).
A utilização conjunta de agonistas adrenérgicos alfa-2 e tiletamina-zolazepam tem sido recentemente avaliada em felídeos selvagens (DEEM et al., 1998; FIGUEIREDO et al., 2001), não existindo estudos sobre os efeitos em gatos domésticos.

Deste modo, objetivou-se, neste estudo, avaliar os efeitos anestésicos e cardiovasculares da associação da tiletamina-zolazepam e romifidina, e a influência da adição do butorfanol sobre os parâmetros avaliados.

\section{MATERIAL E MÉTODOS}

Foram utilizados 6 gatos, sem raça definida, machos e fêmeas, adultos, castrados, com peso médio de $2,5 \pm 0,67 \mathrm{~kg}$, mantidos em recinto coletivo e provenientes do gatil de experimentação animal do Hospital Veterinário da Universidade de Brasília. Previamente aos tratamentos experimentais, os animais foram submetidos à avaliação clínica, e realizados hemograma, dosagem sérica de creatinina, uréia, proteínas totais, e alanina-aminotransferase no intuito de excluir qualquer animal considerado enfermo. Os animais foram submetidos a jejum sólido de 12 horas e hídrico de 4 horas antes dos procedimentos anestésicos. Os animais foram dispostos em dois grupos: Grupo RTZ, administração de $7 \mathrm{mg} . \mathrm{kg}^{-1}$ de tiletamina e $7 \mathrm{mg} . \mathrm{kg}^{-1} \mathrm{de}$ zolazepam a e $40 \mu \mathrm{g} . \mathrm{kg}^{-1}$ de romifidina ${ }^{\mathrm{b}}$; e Grupo RTZB, administração de $7 \mathrm{mg} \cdot \mathrm{kg}^{-1}$ de tiletamina, $7 \mathrm{mg} \cdot \mathrm{kg}^{-1}$ de zolazepam, $40 \mu \mathrm{g} . \mathrm{kg}^{-1}$ de romifidina e $0,2 \mathrm{mg} . \mathrm{kg}^{-1} \mathrm{de}$ butorfanolc. Os agentes acima citados foram dispensados em seringas individuais, sendo posteriormente misturados e administrados numa mesma seringa pela via intramuscular. Adotou-se intervalo mínimo de três semanas entre a administração de cada associação anestésica.

Por meio de monitor multiparamétrico ${ }^{\mathrm{d}}$, realizaram-se seis aferições consecutivas dos seguintes parâmetros para determinação dos valores basais: pressão arterial sistólica (PAS), diastólica (PAD) e média (PAM) não-invasiva por método oscilométrico e saturação parcial de oxigênio $\left(\mathrm{SpO}_{2}\right)$. A freqüência cardíaca (FC) foi determinada por método auscultativo através de estetoscópio; a freqüência respiratória $(\mathrm{FR})$ foi determinada pelo número de excursões torácicas durante um minuto e a temperatura retal (TR) foi obtida por meio de termômetro digital. Os dados foram computados sendo calculadas as médias e desvios-padrão. Todos os parâmetros foram avaliados no momento anterior à aplicação das associações anestésicas (momento 0) e aos 10, 20 ,30, 40, 50, 60, 90 e 120 minutos após aplicação dos fármacos. 
Avaliou-se o período de latência (intervalo de tempo entre a administração da associação e a perda dos reflexos posturais), período anestésico hábil (tempo compreendido entre o decúbito até a primeira tentativa de levantar a cabeça do solo), e período de recuperação (período entre o fim do tempo anestésico hábil e recuperação da postura quadrupedal). Além destas variáveis, utilizou-se um sistema qualitativo de escore por números (Tabela 1), para avaliação de sedação, miorrelaxamento, analgesia, postura, resposta

Tabela 1 - Critérios adotados para a avaliação dos parâmetros anestésicos em gatos anestesiados com romifidinatiletamina-zolazepam (RTZ) e romifidina-tiletaminazolazepam-butorfanol (RTZB).

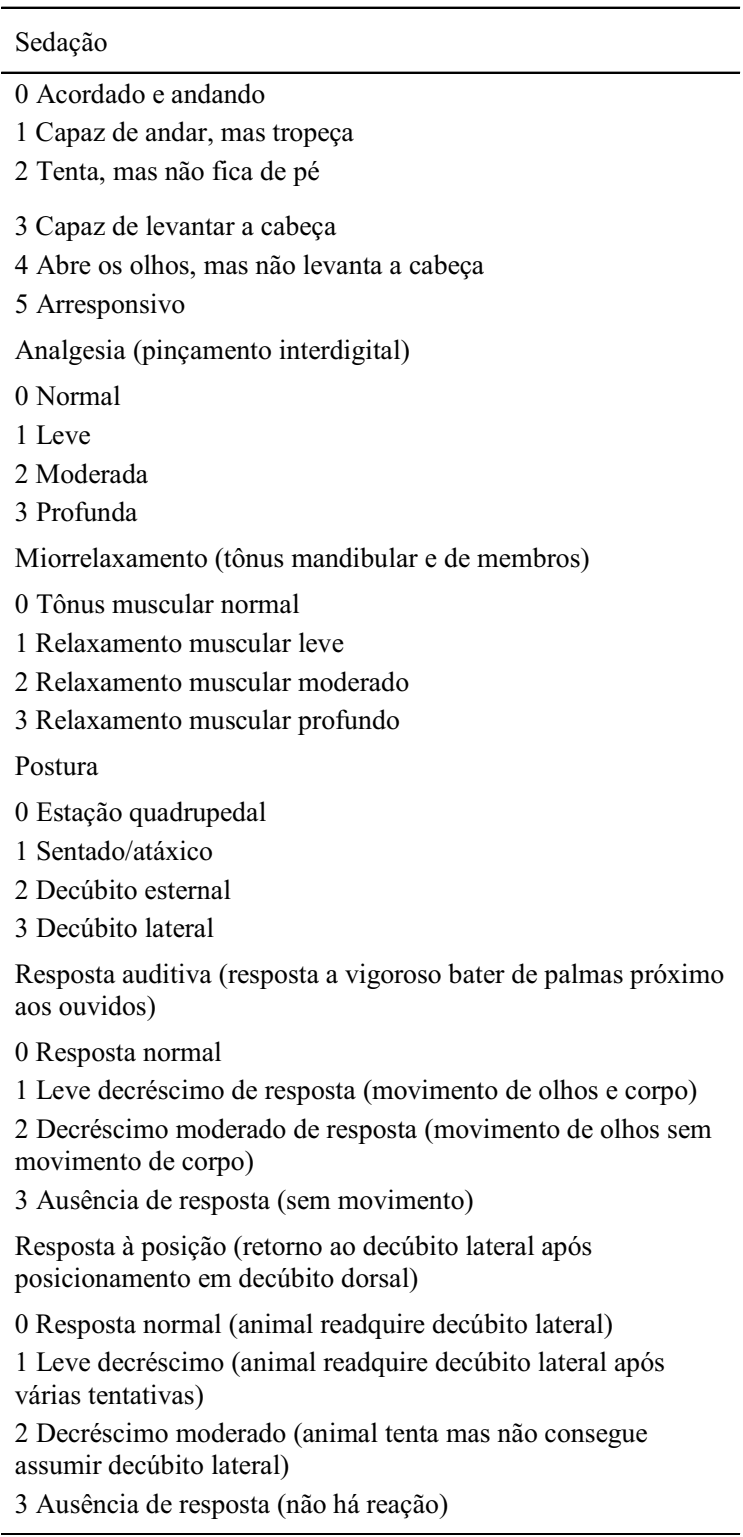

auditiva e resposta à posição, conforme descrito por LEMKE (1999).

Os dados paramétricos foram avaliados utilizando a ANOVA para análises repetidas seguida pelo teste de Student-Newman-Keuls para comparação das diferenças entre os tempos em cada grupo e entre os grupos ${ }^{\mathrm{e}}$. Os escores de sedação, miorelaxamento, analgesia, postura, resposta auditiva e resposta à posição foram analisados pelo teste de Friedman $^{\mathrm{e}}$, para comparação dos valores entre os grupos, sendo expressos em medianas e variação entre 25 e $75 \%$. O período de latência, período anestésico hábil e período de recuperação foram comparados entre os grupos pelo teste t de Student. As diferenças foram consideradas estatisticamente significativas quando $\mathrm{P}<0,05$.

\section{RESULTADOS}

A adição do butorfanol à associação tiletamina-zolazepam/romifidina resultou em significativa redução no período de latência e aumento no período anestésico hábil, sem contudo alterar o período de recuperação dos animais. Os intervalos de tempo nos grupos RTZ e RTZB estão dispostos na tabela 1 .

Não foram observadas diferenças entre os grupos antes da administração das associações anestésicas para nenhum parâmetro. Diferente do grupo RTZ, no qual houve manutenção da freqüência cardíaca durante os primeiros 60 minutos de anestesia, observouse decréscimo da freqüência cardíaca no grupo RTZB a partir de 10 minutos após a administração da associação anestésica. Apesar da queda da freqüência cardíaca observada no grupo RTZB, a partir dos 10 minutos após a administração da associação anestésica, não foram observadas diferenças estatísticas entre os grupos. De maneira geral, a pressão arterial em ambos os grupos permaneceu estável, existindo acréscimo

Tabela 2 - Médias e desvios-padrão dos períodos de latência, período anestésico hábil e período de recuperação em gatos anestesiados com romifidina-tiletaminazolazepam (RTZ) ou romifidina-tiletaminazolazepam-butorfanol (RTZB).

\begin{tabular}{lcc}
\hline \multirow{2}{*}{ PARÂMETRO } & \multicolumn{2}{c}{ GRUPO } \\
\cline { 2 - 3 } & RTZ & RTZB \\
\hline Período de latência & $7,21 \pm 1,0^{*}$ & $5,55 \pm 2,0^{*}$ \\
Período anestésico hábil & $101,06 \pm 48,3^{*}$ & $167,27 \pm 101,4^{*}$ \\
Período de recuperação & $28,47 \pm 40,6$ & $17,11 \pm 27,9$ \\
\hline
\end{tabular}

* Valor significativamente diferente entre os grupos $(\mathrm{P}<0.05)$.

Ciência Rural, v. 33, n. 6, nov-dez, 2003. 
significativo da PAS aos 10, 30 e 60 minutos no grupo RTZ. Os valores de PAS apresentaram-se estatisticamente diferentes após 60 minutos da administração das combinações anestésicas, entretanto não foram observadas diferenças significativas entre os valores de PAD e PAM entre os grupos durante todo o período de avaliação.

Ambos os protocolos promoveram diminuição da freqüência respiratória, sendo esta mais prolongada no grupo RTZB. Imediatamente após a administração das combinações anestésicas, a freqüência respiratória apresentou diferenças significativas entre os grupos. Apesar deste achado, não se observou decréscimo dos valores de saturação parcial de oxigênio nos momentos avaliados. A temperatura retal permaneceu estável em ambos os protocolos durante o período de monitoramento. Os valores referentes aos parâmetros vitais analisados se encontram dispostos na tabela 3 .

A adição do butorfanol resultou em significativa diferença nos escores de sedação aos 30 , 50 e 120 minutos de anestesia. A comparação dos escores de analgesia entre os grupos demonstrou diferença estatística apenas aos 90 minutos, quando os animais do grupo RTZB apresentaram valores mais elevados. No entanto, observou-se que a adição de butorfanol à associação romifidina-tiletaminazolazepam promoveu analgesia superior àquela verificada no grupo RTZ (Tabela 4).

\section{DISCUSSÃO}

É indiscutível que a associação de benzodiazepínicos, opióides e agonistas adrenérgicos alfa-2 possibilite a realização de procedimentos de curta duração (TRANQUILLI et al., 1990; VERSTEGEN \& PETCHO, 1993; PYPENDOP et al., 1996). Apesar deste fato, grande parte dos estudos é realizada em cães, sendo os conhecimentos obtidos normalmente extrapolados para os gatos, os quais apresentam diversas particularidades com relação à biotransformação e resposta frente aos agentes anestésicos, entre eles os opióides (EVANS, 1988).

A associação de agonistas adrenérgicos alfa2 à tiletamina-zolazepam resulta em importante decréscimo de freqüência cardíaca (WILSON et al., 1993; DEEM et al., 1998), fato observado somente após 90 minutos de anestesia no grupo RTZ, o que indica predomínio da ação cardioestimulatória da tiletamina em grande parte do tempo de anestesia (HELLYER et al., 1989 ;WILSON et al., 1993). A utilização de butorfanol associado à tiletamina-zolazepam promove

Tabela 3 - Médias e desvios-padrão dos valores de freqüência cardíaca (FC), pressão arterial sistólica (PAS), diastólica (PAD), média (PAM), freqüência respiratória (FR), saturação parcial de oxihemoglobina $\left(\mathrm{SpO}_{2}\right)$ e temperatura retal (TR) em gatos anestesiados com romifidina-tiletamina-zolazepam (RTZ) ou romifidina-tiletamina-zolazepam-butorfanol (RTZB).

\begin{tabular}{|c|c|c|c|c|c|c|c|c|}
\hline Grupo & Tempo & $\mathrm{FC}$ & PAS & PAD & PAM & FR & $\mathrm{SpO}_{2}$ & TR \\
\hline \multirow[t]{10}{*}{ RTZ } & Basal & $162,6 \pm 28,1$ & $105,5 \pm 5,3$ & $82,8 \pm 9,8$ & $90,1 \pm 6,1$ & $48,3 \pm 11,6$ & $96,5 \pm 1,3$ & $38,6 \pm 0,2$ \\
\hline & 5 & $160,3 \pm 21,9$ & $111,0 \pm 11,5$ & $71,0 \pm 13,9$ & $87,5 \pm 13,4$ & $32,6 \pm 8,5^{*, \mathbf{a}}$ & $96,0 \pm 0,6$ & $38,9 \pm 0,5$ \\
\hline & 10 & $152,3 \pm 16,1$ & $119,5 \pm 14,7^{*}$ & $79,5 \pm 15,4$ & $96,6 \pm 15,0$ & $30,0 \pm 9,2^{*}$ & $96,3 \pm 1,2$ & $38,9 \pm 0,7$ \\
\hline & 20 & $146,8 \pm 21,7$ & $118,3 \pm 11,6$ & $80,8 \pm 14,4$ & $97,5 \pm 11,9$ & $31,3 \pm 6,4^{*}$ & $96,5 \pm 1,3$ & $39,0 \pm 0,7$ \\
\hline & 30 & $146,3 \pm 16,9$ & $121,1 \pm 8,7^{*}$ & $87,1 \pm 17,8$ & $100,1 \pm 13,0$ & $29,3 \pm 7,8^{*}$ & $95,1 \pm 1,4$ & $39,0 \pm 0,8$ \\
\hline & 40 & $143,6 \pm 26,2$ & $121,4 \pm 11,8$ & $80,2 \pm 14,5$ & $98,0 \pm 11,3$ & $29,6 \pm 5,8^{*}$ & $96,8 \pm 1,6$ & $39,2 \pm 0,6$ \\
\hline & 50 & $139,6 \pm 27,8$ & $117,0 \pm 12,0$ & $79,6 \pm 12,3$ & $96,0 \pm 8,7$ & $31,6 \pm 6,8^{*}$ & $96,6 \pm 1,5$ & $39,0 \pm 0,6$ \\
\hline & 60 & $131,5 \pm 11,1$ & $127,5 \pm 9,0^{*}, \mathbf{a}$ & $81,2 \pm 12,1$ & $94,0 \pm 15,8$ & $33,5 \pm 7,1$ & $96,5 \pm 1,2$ & $39,1 \pm 0,7$ \\
\hline & 90 & $131,0 \pm 9,3^{*}$ & $112,0 \pm 8,3$ & $69,3 \pm 14,1$ & $80,6 \pm 19,6$ & $47,0 \pm 15,7$ & $97,6 \pm 0,5$ & $38,6 \pm 0,7$ \\
\hline & 120 & $123,3 \pm 10,0^{*}$ & $106,3 \pm 14,0$ & $87,0 \pm 5,6$ & $97,5 \pm 6,3$ & $54,6 \pm 14,4^{\mathrm{a}}$ & $96,0 \pm 2,8$ & $38,7 \pm 0,4$ \\
\hline \multirow[t]{10}{*}{ RTZB } & Basal & $169,3 \pm 29,2$ & $105,1 \pm 10,4$ & $76,8 \pm 19,0$ & $86,5 \pm 13,1$ & $55,0 \pm 7,1$ & $97,3 \pm 0,5$ & $38,9 \pm 0,3$ \\
\hline & 5 & $144,1 \pm 23,8$ & $107,6 \pm 10,7$ & $78,0 \pm 13,3$ & $89,0 \pm 10,0$ & $50,6 \pm 28,3^{* \mathbf{a}}$ & $97,1 \pm 1,1$ & $38,9 \pm 0,8$ \\
\hline & 10 & $137,6 \pm 23,5^{*}$ & $112,6 \pm 11,6$ & $71,0 \pm 14,2$ & $88,0 \pm 13,7$ & $33,3 \pm 11,8^{*}$ & $95,5 \pm 3,0$ & $38,8 \pm 1,0$ \\
\hline & 20 & $134,8 \pm 24,2^{*}$ & $110,0 \pm 19,3$ & $70,3 \pm 19,3$ & $84,6 \pm 20,9$ & $31,6 \pm 8,3^{*}$ & $96,6 \pm 1,5$ & $38,9 \pm 1,0$ \\
\hline & 30 & $130,5 \pm 23,4^{*}$ & $111,0 \pm 16,7$ & $76,5 \pm 14,0$ & $90,3 \pm 12,9$ & $31,0 \pm 8,3^{*}$ & $96,0 \pm 2,0$ & $38,9 \pm 0,8$ \\
\hline & 40 & $132,6 \pm 20,6^{*}$ & $108,8 \pm 10,6$ & $78,1 \pm 12,6$ & $91,0 \pm 9,5$ & $32,6 \pm 9,1^{*}$ & $96,6 \pm 2,2$ & $38,9 \pm 0,7$ \\
\hline & 50 & $126,1 \pm 19,8^{*}$ & $115,6 \pm 5,8$ & $78,3 \pm 18,4$ & $91,6 \pm 15,2$ & $33,1 \pm 11,5^{*}$ & $96,5 \pm 1,7$ & $38,9 \pm 0,8$ \\
\hline & 60 & $124,3 \pm 18,9^{*}$ & $108,5 \pm 13,1^{\mathrm{a}}$ & $69,0 \pm 18,7$ & $84,8 \pm 18,4$ & $35,6 \pm 11,5^{*}$ & $96,3 \pm 1,6$ & $38,8 \pm 0,7$ \\
\hline & 90 & $129,2 \pm 26,2^{*}$ & $106,5 \pm 5,4$ & $61,7 \pm 11,5$ & $79,2 \pm 11,5$ & $31,5 \pm 6,1^{*}$ & $96,7 \pm 1,8$ & $38,6 \pm 0,5$ \\
\hline & 120 & $114,2 \pm 23,2^{*}$ & $98,5 \pm 7,8$ & $61,0 \pm 11,3$ & $77,5 \pm 8,5$ & $33,0 \pm 9,0^{*}, \mathbf{a}$ & $98,0 \pm 2,1$ & $38,6 \pm 0,6$ \\
\hline
\end{tabular}

* Valor significativamente diferente do valor basal. ${ }^{a}$ Valor significativamente diferente entre os grupos $(\mathrm{P}<0.05)$. 
Tabela 4 - Medianas dos escores de sedação (SED), postura (POS), resposta à posição (RPS), miorrelaxamento (MIO), analgesia (ANG) e resposta auditiva (AUD) em gatos anestesiados com romifidina-tiletamina-zolazepam (RTZ) ou romifidina-tiletamina-zolazepambutorfanol (RTZB)

\begin{tabular}{|c|c|c|c|c|c|c|c|}
\hline Grupo & Tempo & SED & POS & RPS & MIO & ANG & AUD \\
\hline \multirow{20}{*}{ RTZ } & \multirow{2}{*}{ Basal } & 0,0 & 0,0 & 0,0 & 0,0 & 0,0 & 0,0 \\
\hline & & $(0-0)$ & $(0-0)$ & $(0-0)$ & $(0-0)$ & $(0-0)$ & $(0-0)$ \\
\hline & \multirow{2}{*}{5} & 4,0 & 3,0 & 2,5 & 2,5 & 2,0 & 3,0 \\
\hline & & $(3,4-4,0)$ & $(2,5-3,0)$ & $(2,0-3,2)$ & $(2,1-3,0)$ & $(1,5-2,0)$ & $(2,5-3,0)$ \\
\hline & \multirow{2}{*}{10} & 4,0 & 3,0 & 3,0 & 2,5 & 2,0 & 3,0 \\
\hline & & $(3,5-4,0)$ & $(2,5-3,5)$ & $(3,0-4,0)$ & $(2,5-3,0)$ & $(2,0-2,5)$ & $(3,0-4,0)$ \\
\hline & \multirow{2}{*}{20} & 4,0 & 3,0 & 2,5 & 2,5 & 2,5 & 2,5 \\
\hline & & $(4,0-4,5)$ & $(3,0-3,5)$ & $(2,5-3,3)$ & $(2,5-3,0)$ & $(2,0-2,5)$ & $(2,5-3,0)$ \\
\hline & \multirow{2}{*}{30} & $3,5^{*}$ & $2,5^{*}$ & 2,5 & 2,5 & 2,5 & 2,5 \\
\hline & & $(3,0-4,3)$ & $(2,0-2,5)$ & $(2,5-3,0)$ & $(2,5-3,0)$ & $(2,0-2,5)$ & $(2,0-3,0)$ \\
\hline & \multirow{2}{*}{40} & 4,0 & 3,0 & 3,0 & 2,5 & 3,0 & 3,0 \\
\hline & & $(4,0-4,5)$ & $(3,0-4,0)$ & $(2,5-3,0)$ & $(2,5-3,0)$ & $(2,5-3,0)$ & $(3,0-3,5)$ \\
\hline & \multirow{2}{*}{50} & $3,5^{*}$ & 2,5 & 2,5 & 2,5 & 2,0 & 2,0 \\
\hline & & $(3,0-4,0)$ & $(2,5-3,0)$ & $(2,1-3,0)$ & $(2,5-3,0)$ & $(2,0-2,5)$ & $(2,0-2,5)$ \\
\hline & \multirow{2}{*}{60} & 5,0 & 3,0 & 3,0 & 3,0 & 1,5 & 3,0 \\
\hline & & $(4,0-5,0)$ & $(2,5-3,0)$ & $(2,5-3,0)$ & $(2,5-3,0)$ & $(1,5-2,0)$ & $(2,5-3,5)$ \\
\hline & \multirow[b]{2}{*}{90} & 4,0 & 3,0 & 2,0 & 2,0 & $0,5^{*}$ & 2,0 \\
\hline & & $(4,0-5,0)$ & $(2,5-3,5)$ & $(2,0-2,5)$ & $(2,0-2,5)$ & $(0,5-1,2)$ & $(2,0-2,5)$ \\
\hline & \multirow{2}{*}{120} & $1,5^{*}$ & 2,5 & $1,5^{*}$ & $1,5^{*}$ & 1,0 & 1,5 \\
\hline & & $(1,0-2,0)$ & $(2,5-3,0)$ & $(1,0-2,0)$ & $(1,5-2,0)$ & $(1,0-1,2)$ & $(1,5-2,0)$ \\
\hline \multirow{20}{*}{ RTZB } & \multirow{2}{*}{ Basal } & 0,0 & 0,0 & 0,0 & 0,0 & 0,0 & 0,0 \\
\hline & & $(0-0)$ & $(0-0)$ & $(0-0)$ & $(0-0)$ & $(0-0)$ & $(0-0)$ \\
\hline & \multirow{2}{*}{5} & 4,5 & 3,0 & 2,5 & 2,0 & 1,5 & 2,0 \\
\hline & & $(4,0-4,5)$ & $(2,5-3,0)$ & $(2,0-3,0)$ & $(2,0-3,0)$ & $(1,5-2,0)$ & $(2,0-2,5)$ \\
\hline & \multirow{2}{*}{10} & 5,0 & 3,0 & 3,0 & 3,0 & 3,0 & 3,0 \\
\hline & & $(4,0-5,0)$ & $(3,0-4,0)$ & $(3,0-4,0)$ & $(2,5-3,0)$ & $(2,5-3,0)$ & $(3,0-4,0)$ \\
\hline & \multirow{2}{*}{20} & 5,0 & 3,0 & 3,0 & 3,0 & 3,0 & 3,0 \\
\hline & & $(4,5-5,0)$ & $(2,9-3,5)$ & $(3,0-4,1)$ & $(2,5-3,0)$ & $(2,5-3,0)$ & $(30-4,0)$ \\
\hline & \multirow{2}{*}{30} & $5,0^{*}$ & $3,0^{*}$ & 3,0 & 3,0 & 3,0 & 3,0 \\
\hline & & $(4,5-5,0)$ & $(3,0-3,5)$ & $(30-4,0)$ & $(2,5-3,0)$ & $(2,0-3,0)$ & $(3,0-3,5)$ \\
\hline & \multirow{2}{*}{40} & 5,0 & 3,0 & 3,0 & 3,0 & 3,0 & 3,0 \\
\hline & & $(4,5-5-0)$ & $(3,0-4,0)$ & $(3,0-3,5)$ & $(2,5-3,0)$ & $(2,5-3,0)$ & $(3,0-3,5)$ \\
\hline & \multirow{2}{*}{50} & $5,0^{*}$ & 3,0 & 2,5 & 3,0 & 2,5 & 3,0 \\
\hline & & $(4,5-5,0)$ & $(3,3-4,0)$ & $(2,5-3,5)$ & $(2,5-3,0)$ & $(2,5-3,0)$ & $(2,5-3,0)$ \\
\hline & \multirow{2}{*}{60} & 4,5 & 3,0 & 3,0 & 3,0 & 2,0 & 2,0 \\
\hline & & $(4,5-5,0)$ & $(2,5-3,0)$ & $(2,5-3,0)$ & $(2,5-3,0)$ & $(2,0-2,5)$ & $(2,0-2,5)$ \\
\hline & \multirow{2}{*}{90} & 5,0 & 3,0 & 3,0 & 3,0 & $2,5^{*}$ & 2,5 \\
\hline & & $(4,0-5,0)$ & $(2,5-3,0)$ & $(2,5-3,5)$ & $(2,5-3,5)$ & $(2,0-2,5)$ & $(2,0-2,5)$ \\
\hline & \multirow{2}{*}{120} & $5,0^{*}$ & 3,0 & $3,0^{*}$ & $3,0^{*}$ & 2,0 & 2,0 \\
\hline & & $(4,0-5,0)$ & $(2,5-3,0)$ & $(2,5-3,5)$ & $(2,5-3,5)$ & $(1,5-2,0)$ & $(2,0-2,5)$ \\
\hline
\end{tabular}

*Valor significativamente diferente entre os grupos $(\mathrm{P}<0.05)$. Os valores entre parênteses representam a variação de escores presente entre 25 e $75 \%$ dos indivíduos do grupo.

discreta e transitória bradicardia (WILSON et al., 1993), ressaltando seus mínimos efeitos deletérios (TRIM, 1983). Apesar deste fato, a adição do butorfanol ao protocolo resultou em diminuição da freqüência cardíaca, provavelmente pela facilitação do tono parasimpático associado aos agonistas adrenérgicos alfa-2, sendo que, contrariamente ao grupo RTZ, a ação simpática da tiletamina pode não ter sido eficaz na prevenção da bradicardia induzida pela associação do butorfanol a romifidina, conforme relataram SELMI et al. (2001).

Diferentemente dos resultados em guepardos, nos quais a administração de medetomidina e tiletamina-zolazepam promoveu significativa

Ciência Rural, v. 33, n. 6, nov-dez, 2003. 
hipertensão arterial (DEEM et al., 1998), em gatos domésticos, a associação da romifidina e tiletaminazolazepam não resultou em alteração dos valores de pressão arterial. Os aumentos significativos da PAS em alguns momentos, neste grupo, evidenciam resultados anteriores (HELLYER et al., 1989). Em cães, a administração de benzodiazepínicos, opióides e agonistas adrenérgicos alfa- 2 pode resultar em hipertensão arterial (GROSS et al., 1990; ITAMOTO et al., 2000). A administração de medetomidina e butorfanol em cães atropinizados promoveu aumento da pressão arterial, entretanto este aumento decresceu prontamente aos valores normais após a administração de cetamina, (TOMIZAWA et al., 1997). Apesar do decréscimo da PAS e da PAD em alguns momentos da avaliação, de forma não significativa, a associação do butorfanol a romifidina-tiletamina-zolazepam demonstrou mínimas influências sobre a pressão arterial. Estudos recentes têm mostrado que o uso isolado de romifidina, ou sua associação com o butorfanol, não promoveu alterações na pressão arterial de gatos (SELMI et al., 2001). Desta forma, a associação de RTZ isoladamente ou em associação ao butorfanol não causou alterações significativas da pressão arterial, uma vez que a PAM se manteve dentro de valores normais para a espécie estudada.

Estudos recentes em gatos demonstraram que a frequiência respiratória não é alterada pela administração de romifidina ou pela associação de romifidina e butorfanol (SELMI et al. 2001). Entretanto, observouse decréscimo transitório na freqüência respiratória após a administração de RTZ, possivelmente pela ação dos agonistas adrenérgicos alfa-2 sobre o centro respiratório (HALL \& CLARKE, 1991) em combinação com a TZ. Apesar da discreta depressão respiratória produzida pelo butorfanol, um agonista kappa (HALL \& CLARKE, 1991), a adição deste fármaco contribuiu para o decréscimo da freqüência respiratória no grupo RTZB de forma mais prolongada, sem que fossem observadas diferenças estatísticas entre os grupos, na maior parte deste estudo. A diferença significativa dos valores médios de freqüência respiratória, observada entre os grupos após a administração dos fármacos, é justificada pela maior média deste parâmetro no grupo RTZB, apesar desta diferença não ser estatisticamente significativa no momento que antecedeu os tratamentos. A adição de romifidina e romifidina-butorfanol à tiletaminazolazepam não alterou significativamente os valores de saturação parcial de oxigênio, o que sugere a relativa segurança de ambos os protocolos empregados sobre os parâmetros respiratórios. Entretanto, estudos relativos às pressões parciais de gases sangüíneos poderiam melhor elucidar tal questão.
Em gatos, a utilização de romifidina $\left(100 \mu \mathrm{g} \cdot \mathrm{kg}^{-1}\right)$ ou a associação de romifidina $\left(40 \mu \mathrm{g} \cdot \mathrm{kg}^{-1}\right)$ e butorfanol $\left(0,2 \mathrm{mg} \cdot \mathrm{kg}^{-1}\right)$ não produziu diferenças entre os períodos de latência ou anestésico hábil, entretanto, as doses utilizadas de romifidina apresentavam variação entre os grupos, sugerindo que uma menor dose de romifidina, em associação ao butorfanol, tivesse o mesmo efeito sedativo que uma dose maior de romifidina, em uso isolado (SELMI et al., 2001). Desta forma, as diferenças observadas nos períodos de latência e anestésico hábil, entre os grupos deste estudo, empregando doses similares de romifidina e tiletamina- zolazepam, podem ser imputadas a um maior efeito sinérgico da associação romifidinabutorfanol quando comparada ao uso isolado da romifidina.

A adição do butorfanol a RTZ em gatos proporcionou, apesar de não serem significativamente diferentes, aumento do grau de sedação e miorrelaxamento, resultando em prolongamento do tempo anestésico hábil. Apesar do butorfanol promover analgesia mais duradoura ao protocolo anestésico, não foram observadas marcantes diferenças de analgesia entre os grupos durante o período de avaliação. Estudos clínicos demonstram que o butorfanol produz analgesia essencialmente visceral com menor ação somática (WAGNER, 1999). Acredita-se que, em razão da avaliação de analgesia neste trabalho ter sido essencialmente somática e de forma subjetiva, deva ser melhor estudada a eventual ação antinociceptiva da adição do butorfanol ao protocolo.

A administração de tiletamina-zolazepam está relacionada à ocorrência de recuperação anestésica conturbada (ILKIW, 1992; HUBBELL, 2000). Atribuise a recuperação anestésica mais tranqüila à adição da romifidina ou romifidina-butorfanol, visto que em ambos os grupos não foram observados quaisquer incidentes durante a recuperação.

Conclui-se, com base nos resultados apresentados, que a associação de romifidina e tiletamina-zolazepam resulta em adequada qualidade anestésica, tendo como principal efeito deletério o transitório decréscimo da freqüência respiratória. A adição do butorfanol à combinação RTZ promove diminuição das freqüências cardíaca e respiratória de forma mais duradoura, contudo propiciando analgesia mais duradoura.

\footnotetext{
a - Telazol, Fort Dodge, Iowa, USA

b - Sedivet, Boehringer, Brasil.

c - Torbugesic, Fort Dodge Laboratories, Inc., USA.

d - Dixtal - DX 2710, Brasil.

e - Graphpad Instat. V.3.00 for Windows 95, GraphPad Software, California USA.
} 


\section{REFERÊNCIAS BIBLIOGRÁFICAS}

CALDERWOOD, H.W. et al. Cardiorespiratory effects of tiletamine in cats. Am J Vet Res, v.32, n.10, p.1511-1515, 1971.

CRUZ, M.L. et al. A preliminary trial comparison of several anesthetic techniques in cats. Can Vet J, v.41, n.6, p.481-485, 2000 .

DEEM, S.L.; KO, J.C.; CINTINO, S.B. Anesthetic and cardiorespiratory effects of tiletamine-zolazepam-medetomidine in cheetahs. J Am Vet Med Assoc, v.213, n.7, p.1022-1026, 1998.

EVANS, R.J. Terapêutica. In: CHANDLER, E.A.; HILBERY, A.D.R.; GASKELL, C.J. (Ed). Medicina e terapêutica de felinos. 2. ed. São Paulo : Manole, 1988. p.419-436.

FAGGELLA, A.M.; ARONSOHN, M.G. Anesthetic techniques for 6- to 14-week-old kittens. J Am Vet Med Assoc, v.202, n.1, p.56-62, 1993.

FIGUEIREDO, J.P. et al. Efeitos de tiletamina-zolazepam e romifidina em tigres-de-bengala (Panthera tigris tigris) prémedicados com atropina. Arch Vet Sci, v.6, Sup1, p.16, 2001.

GROSS, M.E. et al. Hemodynamic effects of intravenous midazolam-xylazine-butorphanol in dogs. Vet Surg, v.19, n.2 p.173-180, 1990.

HALL, L.W.; CLARKE, K.W. Principles of sedation, analgesia and premedication. In: HALL, L.W.; CLARKE, K.W. (Ed) Veterinary anesthesia. 9. ed. London : Bailliere Tindall, 1991. p. 51-79.

HELLYER, P.; MUIR, W.W.; HUBBELL, J.A.E. Cardiorespiratory effects of the intravenous administration of tiletamine-zolazepam to cats. Vet Surg, v.17, n.2, p.105-109, 1988.

HELLYER, P. et al. Cardiorespiratory effects of tiletaminezolazepam to dogs. Vet Surg, v.18, n.2, p.160-165, 1989.

HOSGOOD, G. Pharmacology features of butorphanol in dogs and cats. J Am Vet Med Assoc, v.196, n.1, p.135-136, 1990

HUBBELL, J.A. Pratical methods of anesthesia. In: BICHARD, S.J.; SHERDING, R.G. (Ed). Saunders manual of small animal pratice. 2. ed. Philadelphia : Saunders, 2000. p.12-20.

ILKIW, J.E. Other potentially usefull new injectable anesthetic agents. Opinions in small animal anesthesia. Vet Clin N Am Smal Anim Pract, v.22, n.2, p.281-289, 1992
ITAMOTO, K. et al. Anesthetic and cardiopulmonary effects of balanced anesthesia with medetomidine-midazolam and butorphanol in dogs. J Vet Med, v.47, n.7, p.411-420, 2000

KO, J.C. et al. Comparison of sedative and cardiorespiratory effects of medetomidine and medetomidine-butorphanol combination in dogs. Am J Vet Res, v.57, n.4, p.535-540, 1996.

LEMKE, K.A. Sedative effects of intramuscular administration of a low dose of romifidine in dogs. Am J Vet Res, v.60, n.2, p.162-168, 1999

SELMI, A.L. et al. Efeitos cardiovasculares e sedativos da associação romifidina/butorfanol em gatos pré-tratados ou não pela atropina. Cie Anim, v.10, n.3, p.144, 2001.

PYPENDOP, B.; SERTEYN, D.; VERSTEGEN, J. Hemodynamic effects of medetomidine-midazolam-butorphanol and medetomidine-midazolam-buprenorphine combinations and reversibility by atipamezole in dogs. Am J Vet Res, v.57, n.5, p.724-730, 1996.

PYPENDOP, B.H.; VERSTEGEN, J.P. Cardiovascular effects of romifidine in dogs. Am J Vet Res, v.62, n.4, p.490-495, 2001

TOMIZAWA, N. et al. A comparative study of medetomidinebutorphanol and medetomidine-ketamine anaesthesia in dogs. Zentralb Vet Reihe, v.44, n.4, p.189-194, 1997.

TRANQUILLI, W.J. et al. Evaluation of three midazolam-xylazine mixtures. Preliminary trials in dogs. Vet Surg, v.19, n.2, p.168$172,1990$.

TRIM, C.M. Cardiopulmonary effects of butorphanol tartrate in dogs. Am J Vet Res, v.44, n.2, p.329-331, 1983.

VERSTEGEN, J.; PETCHO, A. Medetomidine-butorphanolmidazolam for anesthesia in dogs and its reversal by atipamezole. Vet Rec, v.132, p.353-357, 1993

WAGNER, A.E. Is butorphanol analgesic in dogs and cats? Vet Med, v.94, n.4, p.346-351, 1999.

WILSON, R.P. et al. Antinociceptive properties of tiletamine-zolazepam improved by addition of xylazine or butorphanol. Pharm Biochem Behav, v.43, n.4, p.11291133,1992

WILSON, R.P. et al. Cardiovascular and respiratory effects of tiletamine-zolazepam. Pharm Biochem Behav, v.44, n.1, p.1-8, 1993 